\title{
Relação entre os níveis de atividade física e qualidade de vida no uso de drogas em adolescentes
}

\author{
Bruno de Oliveira Pinheiro ${ }^{1}$ \\ André Luiz Monezi Andrade ${ }^{2}$ \\ Denise De Micheli ${ }^{3}$
}

Devido ao crescente consumo de drogas entre os adolescentes em fase escolar, este estudo teve por objetivo avaliar a real contribuição das atividades físicas como método de prevenção ao uso de entorpecentes, considerando os índices de qualidade de vida e padrões de consumo dessas substâncias. O estudo foi realizado com 754 adolescentes entre 14 e 18 anos de idade. Verificou-se, entre os adolescentes com alto nível de atividade física, maiores índices de consumo de substâncias psicotrópicas, bebidas energéticas, substâncias ergogênicas e baixos índices de qualidade de vida. De modo geral, sugere-se a realização de intervenções de caráter educativo sobre o uso de substâncias em geral, tanto para adolescentes praticantes de atividades físicas quanto para familiares e treinadores.

Descritores: Adolescente; Atividade Motora; Qualidade de Vida; Transtornos Relacionados ao Uso de Substâncias.

\footnotetext{
${ }^{1}$ MSc, Professor, Governo do Estado de São Paulo, Secretaria da Educação do Estado de São Paulo, São Paulo, SP, Brasil. ${ }^{2} \mathrm{PhD}$, Professor, Universidade Federal de São Paulo, São Paulo, SP, Brasil.

${ }^{3} \mathrm{PhD}$, Professor Adjunto, Universidade Federal de São Paulo, São Paulo, SP, Brasil.
} 


\title{
Relationship between levels of physical activity and quality of life in drug use in teenagers
}

\begin{abstract}
Due to the increasing drug use among teenagers, this study aimed to evaluate the actual contribution of physical activities as a method of preventing drug use, considering the quality of life indices and patterns of consumption of these substances. The study was conducted with 754 teenagers between 14 and 18 years old. High consumption rates of psychotropic substances, energy drinks, ergogenic substances and low levels of quality of life were found among teenagers with high levels of physical activity. In general, carrying out educational interventions on substance use in general is suggested, for teenagers who practice physical activity, their families and coaches.
\end{abstract}

Descriptors: Adolescent; Motor Activity; Quality of Life; Substance; Related Disorders.

\section{Relación entre los niveles de actividad física y calidad de vida en el uso de drogas en adolescentes}

\begin{abstract}
Debido al creciente consumo de drogas entre los adolescentes en fase escolar, este estudio tuvo por objetivo evaluar la real contribución de las actividades físicas como método de prevención al uso de drogas, considerando los índices de calidad de vida y calidades de consumo de esas substancias. El estudio fue realizado con 754 adolescentes entre 14 y 18 años de edad. Se verificó, entre los adolescentes con alto nivel de actividad física, mayores índices de consumo de substancias psicotrópicas, bebidas energéticas, substancias ergogênicas y bajos índices de calidad de vida. De modo general, se sugiere la realización de intervenciones de carácter educativo sobre el uso de substancias en general, tanto para adolescentes practicantes de actividades físicas cuanto para familiares y entrenadores.
\end{abstract}

Descriptores: Adolescentes; Actividad Motora; Calidad de Vida; Trastornos Relacionados con Sustancias.

Introdução

A prática de atividade física pela população tornou-se uma questão de saúde ainda na década de 1990, de modo que muitos estudos enfatizam a importância da prática de exercícios desde a infância, para a construção de uma vida adulta com hábitos saudáveis ${ }^{(1)}$. Na adolescência, paralelamente à boa nutrição, a atividade física é importante contribuição para o aprimoramento e desenvolvimento do indivíduo, podendo aperfeiçoar o potencial físico para um aproveitamento melhor de suas possibilidades, da mesma maneira que para diminuição dos riscos de futuras doenças ${ }^{(2)}$.

De modo geral, a grande preocupação com a imagem corporal é o estímulo propulsor que leva muitos adolescentes a buscar atividades desportivas. O interesse na promoção da saúde aparece como motivo secundário pelo qual os adolescentes buscam a atividade física em academias, clubes e outros ${ }^{(3)}$. 0 esporte encarado como única ou principal atividade do adolescente pode trazer prejuízos devido à 
desmesurada pressão em relação a um esforço físico exagerado, provocando ansiedade resultante das competições e treinamentos, que pode levá-lo a desequilíbrio com relação a suas satisfações físicas, emocionais e sociais ${ }^{(4)}$.

São verificadas duas tendências quanto à conceptualização do termo qualidade de vida na área da saúde: qualidade de vida como conceito mais genérico e qualidade de vida relacionada com a saúde ${ }^{(5)}$. A saúde percebida é denominada "Qualidade de Vida Relacionada com a Saúde" (QVRS /HealthRelated Quality of Life - HRQOL) e é descrita como um constructo que engloba componentes do bemestar e funções físicas, emocionais, mentais, sociais e comportamentais, como são percebidos pelos próprios indivíduos e pelos outros. Ravens-Sieberer e o grupo Europeu KIDSCREEN( ${ }^{(6)}$ defendem que a conceptualização de qualidade de vida relacionada com a saúde implica um modelo compreensivo de saúde subjetivo e multidimensional. Os autores salientam que a qualidade de vida relacionada com a saúde pode ser vista como um constructo psicológico que descreve aspectos físicos, psicológicos, mentais, sociais e funcionais do bem-estar.

No que se refere à associação entre qualidade de vida e uso de substâncias, em especial na adolescência, estudos indicam que o consumo de drogas por pais ou familiares, a baixa percepção de apoio parental, ausência de práticas religiosas e menor frequência na prática de atividades físicas, propiciam baixos níveis de qualidade de vida e por conseguinte aumentam a incidência de fatores de risco relacionados ao uso de substâncias psicotrópicas e outros comportamentos de risco(7).

Quanto à adoção de melhor estilo de vida, estudos realizados com adolescentes americanos mostraram que níveis moderados de atividades físicas estavam relacionados com a menor incidência de uso de tabaco e maconha, sugerindo efeitos psicossociais positivos nesta faixa etária( ${ }^{(8)}$. Por outro lado, Dearwater et al. ${ }^{(9)}$, ao avaliarem a associação entre qualidade de vida e comportamentos de risco em adolescentes do sexo masculino, verificaram maior incidência de consumo de álcool entre aqueles com maiores níveis de atividade física e esportiva. Outros estudos, com resultados semelhantes, também mostraram que a prática de atividades físicas e esportivas não necessariamente atua como um fator protetor para o uso de substâncias ${ }^{(10)}$.

Neste sentido, este estudo teve como objetivo investigar a relação entre diferentes níveis de atividade física, qualidade de vida e consumo de substâncias psicotrópicas entre alunos de escolas públicas, analisando a real contribuição da prática esportiva como estratégia de prevenção ao uso de drogas.

\section{Materiais e Métodos}

Fizeram parte deste estudo 754 estudantes com idades entre 14 e 18 anos, de ambos os sexos, cursando entre o $9^{\circ}$ ano do ensino fundamental e o $3^{\circ}$ ano do ensino médio de 4 escolas da rede pública estadual da cidade de Guarulhos, na grande São Paulo, selecionadas por critério de conveniência e pareamento sociodemográfico.

Após o contato inicial com as escolas selecionadas foi realizada a apresentação do estudo à direção de cada unidade, a assinatura do Termo de Consentimento Livre e Esclarecido (TCLE) e foram agendados os horários e dias para aplicação dos questionários junto aos estudantes. As turmas foram selecionadas por critério de conveniência sendo a participação dos estudantes voluntária. A aplicação dos questionários foi feita nas salas de aula, com a presença do professor, e por pesquisadores vinculados a este estudo e duração de 50 minutos.. Após o término, foi oferecida as escolas uma palestra informativa que abordou temas relacionados à qualidade de vida, práticas desportivas e uso de drogas lícitas e ilícitas, incluindo bebidas energéticas e ergogênicos.

Para avaliação do nível de atividade física (AF) em diferentes contextos da vida do indivíduo, utilizouse o International Physical Activity Questionnaire (IPAQ), desenvolvido pela organização Mundial de Saúde e validado para a população brasileira ${ }^{(11)}$. Foi aplicada a versão longa do questionário composta por 27 questões distribuídas em cinco domínios: AF no Trabalho; AF de Locomoção; AF Doméstica; Atividades de Lazer; e Tempo gasto sentado durante a semana e fim de semana.

Com base nas informações fornecidas através do IPAQ, foi empregado escore de equivalentes metabólicos (MET) para cada tipo de AF realizada dos cinco domínios avaliados, que foi multiplicado pela frequência semanal (Dias ou semana) e pelo tempo médio das atividades. Posteriormente, somouse o total de cada domínio e calculou-se o total da AF em METs por semana ${ }^{(12)}$. O equivalente metabólico (MET), é uma unidade utilizada para estimar o gasto metabólico da atividade física, tendo como base o consumo de oxigênio. Um MET equivale à taxa metabólica de repouso de aproximadamente $3,5 \mathrm{ml}$ de $\mathrm{O}_{2}$ por minuto. Quando se exprime o gasto de energia em METs, representa-se o número de vezes 
pelo qual o metabolismo de repouso foi multiplicado durante uma atividade. O American College of Sport Medicine sugere que a unidade MET seja utilizada como método para indicar e comparar a intensidade absoluta e gasto energético de diferentes atividades físicas. Nesse contexto, o conceito de MET é aplicado nas orientações gerais à população em relação ao gasto energético das atividades. Portanto o MET é uma medida da intensidade de esforço ${ }^{(13-14)}$. Como resultado, os participantes foram classificados em três diferentes níveis de intensidade de atividade física: Baixo: Indivíduos que não atingiram níveis de AF adequados para serem incluídos nas categorias dois e três (A seguir); Moderado: Indivíduos com prática de AF intensa em 3 ou mais dias na semana por mais de 20 minutos/dia; ou indivíduos que praticam AF moderada ou caminhadas em 5 ou mais dias na semana por mais de 30 minutos/dia; ou indivíduos que praticam combinações de exercícios físicos intensos e moderados em 5 ou mais dias na semana por mais de 30 minutos/dia, atingindo ao menos 600 $\mathrm{METs}$ na semana. Alto: Indivíduos que praticam AF intensa em 3 ou mais dias na semana, atingindo 1500 METs em uma semana ou; indivíduos que praticam qualquer combinação de AF em intensidade intensa e moderada em 7 dias na semana atingindo ao menos 3000 METs na semana.

Para avaliação do índice de Qualidade de Vida Relacionada à Saúde (QVRS) utilizou-se o questionário KIDSCREEN-52, validado para a população brasileira(15). Este instrumento fornece índices de bem-estar e saúde subjetiva em crianças e adolescentes saudáveis e em condições crônicas. Este estudo utilizou a versão curta do instrumento composta por 27 questões relacionadas à percepção de cinco dimensões de QVRS, a saber: Bemestar físico; Bem-estar psicológico; Autonomia e relacionamento com os pais; Amigos e apoio social; Ambiente escolar. As respostas são formatadas por dimensão, em uma escala likert de um a cinco pontos, buscando identificar a frequência ou intensidade de sentimentos, retroativos a semana anterior a aplicação do questionário. Os índices de cada dimensão são computados considerando as respostas do grupo de questões que compõem essa dimensão e igualmente ponderados. Os escores finais equivalentes a cada dimensão são recodificados em uma escala de medida, com variação entre zero e 100 , sendo zero a menor e 100 a maior percepção do indicador de QVRS da dimensão em questão.

Para avaliação do uso de substâncias utilizouse o Drug Use Screening Inventory (DUSI), validado para a população de adolescentes brasileiros ${ }^{(16)}$. Este estudo empregou apenas a tabela de frequência de uso de substâncias no último mês e a área 1 composta por 15 perguntas que abordam problemas associados ao uso, por exemplo: compulsão ou fissura em usar, sintomas de tolerância, abstinência, ou envolvimento em acidentes sob efeito de álcool e/ ou outras drogas. Com vistas a investigar o consumo de bebidas energéticas e substâncias ergogênicas, utilizadas com o objetivo de melhorar o desempenho esportivo e/ou recuperação após o exercício, estas substâncias foram acrescidas à lista daquelas originalmente avaliadas pelo instrumento (Área 1). As questões do DUSI (Área 1) são respondidas com Sim ou Não, e as respostas afirmativas equivalem a presença de problemas. Para a classificação do padrão de uso de substâncias dos participantes adotou-se os mesmos pontos de corte propostos por ocasião da validação do DUSI, a saber: zero a duas respostas afirmativas na área de uso de substâncias equivale a uso experimental; três a sete respostas afirmativas, representa uso abusivo e mais do que sete respostas afirmativas, corresponde a indícios de dependência. Desta forma, os participantes que não pontuaram na área de uso de substância (Área 1) e que não mencionaram consumo de substâncias no último mês, foram classificados como "Não usuários". Para a classificação dos "Usuários Experimentais" considerou-se o consumo de álcool e/ou tabaco na frequência máxima de uma a duas vezes no mês e/ ou até duas respostas afirmativas na área de uso de substâncias. Para a classificação de " Usuários Abusivos" levou-se em conta o consumo máximo de álcool e/ou tabaco de até nove vezes no último mês; e/ou consumo máximo de substâncias ilícitas de até nove vezes no último mês; e/ou três a sete respostas afirmativas na área de uso de substâncias. Para classificação de dependência, estimou-se o consumo de álcool e/ou tabaco superior a 20 vezes no último mês; e/ou consumo de substâncias ilícitas superior a 10 vezes no último mês; e/ou sete ou mais respostas afirmativas na área de uso de substâncias.

Este estudo foi submetido ao Comitê de Ética em Pesquisa da Universidade Federal de São Paulo ( $N^{\circ}$. 534.640), para sua aprovação, e recebeu o Certificado de Apresentação para Apreciação Ética (CAAE) (№.21956613.6.0000.5505).

Utilizou-se o programa SPSS versão 20.0 para a realização das análises estatísticas. Foram feitas estatísticas descritivas e testes de hipóteses. As variáveis contínuas foram expressas como média e desvio padrão e as variáveis categóricas foram 
expressas como frequência absoluta e relativa. A comparação entre os grupos foi realizada pelo teste $t$ de Student para variáveis contínuas e o teste de qui-quadrado para variáveis categóricas. O nível de significância usado para todos os testes foi $\alpha$ igual a 0,05 .

\section{Resultados}

No que se refere aos dados sociodemográficos não foram aobservadas diferenças entre os grupos. A média de idade dos participantes foi de 15,5 anos, pouco mais da metade da amostra era do sexo feminino $(54,5 \%)$ e cursavam a primeira série do ensino médio (57\%). Considerando-se os níveis de atividade física, destaca-se o maior percentual de meninas no grupo de intensidade moderada, quando comparado aos meninos. Observou-se que 3,5\% da amostra declarou ter alguma deficiência (Locomotora ou cardiorrespiratória) considerada impeditiva para a realização de AF nos moldes convencionais. No entanto, supondo que as escolas participantes deste estudo utilizam modalidades de AF adaptadas a indivíduos com necessidades especiais, estes não foram excluídos. Vale mencionar que este percentual de deficientes representa apenas $10 \%$ do grupo de baixo nível de atividade física (Tabela 1).

Tabela 1 - Dados sociodemográficos da amostra considerando os grupos relativos ao nível de atividade física ( $n=754)$. Guarulhos, SP, Brasil, 2014/2015. Dados expressos em porcentagem.

\begin{tabular}{|c|c|c|c|c|c|}
\hline & $\begin{array}{c}\text { Baixo } \\
(n=247)\end{array}$ & $\begin{array}{c}\text { Moderado } \\
(n=387)\end{array}$ & $\begin{array}{c}\text { Alto } \\
(n=120)\end{array}$ & $\mathrm{F} / \mathrm{X} 2$ & p. \\
\hline Idade & & & & 0,00 & 0,99 \\
\hline Média \pm DP & $15,5 \pm 0,83$ & $15,5 \pm 0,89$ & $15,6 \pm 0,74$ & & \\
\hline Sexo & & & & 8,62 & 0,01 \\
\hline Masculino & $51,0(\mathrm{~N}=126)$ & $40,0(\mathrm{~N}=155)$ & $50,0(\mathrm{~N}=60)$ & & \\
\hline Feminino & $49,0(\mathrm{~N}=121)$ & $60,0(\mathrm{~N}=232)$ & $50,0(\mathrm{~N}=60)$ & & \\
\hline Série & & & & 18,48 & 0,01 \\
\hline Nono Ano & $16,6(\mathrm{~N}=41)$ & $21,7(\mathrm{~N}=84)$ & $12,5(\mathrm{~N}=15)$ & & \\
\hline Colegial I & $63,2(N=156)$ & $52,2(\mathrm{~N}=202)$ & $50,3(\mathrm{~N}=60)$ & & \\
\hline Colegial II & $14,6(\mathrm{~N}=36)$ & $18,9(\mathrm{~N}=73)$ & $25,8(\mathrm{~N}=31)$ & & \\
\hline Colegial III & $5,6(N=14)$ & $7,2(\mathrm{~N}=28)$ & $11,4(\mathrm{~N}=14)$ & & \\
\hline Repetência & & & & 3,85 & 0,14 \\
\hline Sim & $27,9(\mathrm{~N}=69)$ & $21,7(\mathrm{~N}=84)$ & $20,8(\mathrm{~N}=25)$ & & \\
\hline Não & $72,1(\mathrm{~N}=178)$ & $78,3(\mathrm{~N}=303)$ & $79,2(\mathrm{~N}=95)$ & & \\
\hline Deficiência física & & & & 1,57 & 0,45 \\
\hline Sim & $4,0(N=10)$ & $3,6(N=14)$ & $1,7(\mathrm{~N}=2)$ & & \\
\hline Não & $96,0(\mathrm{~N}=227)$ & $96,4(\mathrm{~N}=373)$ & $98,3(\mathrm{~N}=118)$ & & \\
\hline
\end{tabular}

Quanto aos índices de qualidade de vida, observou-se que os adolescentes do grupo de alta intensidade de atividade física apresentaram piores níveis de qualidade de vida (Representado por menores médias) nas dimensões relacionadas à autonomia ou relacionamento com os pais, amigos,apoio social e desempenho escolar, quando comparados aos adolescentes dos demais grupos (Tabela 2). 
Tabela 2 - Associação entre os índices de Qualidade de Vida (QV) e os níveis de atividade física dos adolescentes ( $n=754)$. Guarulhos, SP, Brasil, 2014/2015.

\begin{tabular}{lccc}
\hline & $\begin{array}{c}\text { Baixo } \\
(\mathrm{n}=247)\end{array}$ & $\begin{array}{c}\text { Moderado } \\
(\mathrm{n}=287)\end{array}$ & $\begin{array}{c}\text { Alto } \\
(\mathrm{n}=120)\end{array}$ \\
\hline \multicolumn{1}{c}{ Dimensões de QV } & Média $\pm \mathrm{DP}$ & Média $\pm \mathrm{DP}$ & Média \pm DP \\
\hline Bem-estar físico & $61,0 \pm 14,5$ & $63,5 \pm 12,2$ & $62,8 \pm 14,8$ \\
Bem-estar Psicológico & $62,5 \pm 12,0$ & $64,5 \pm 9,8$ & $63,4 \pm 12,5$ \\
Autonomia ou rel. com os & $69,0^{\mathrm{a}} \pm 19,9$ & $65,0^{\mathrm{a}} \pm 20,6$ & $59,5 \pm 20,6^{\mathrm{b}}$ \\
pais & $74,5^{\mathrm{a}} \pm 21,0$ & $74,0^{\mathrm{a}} \pm 20,4$ & $69,5 \pm 18,9^{\mathrm{b}}$ \\
Amigos ou apoio social & $65,0 \pm 20,0$ & $67,0 \pm 19,8$ & $59,0^{\mathrm{b}} \pm 21,5^{\mathrm{b}}$ \\
\hline
\end{tabular}

DP: desvio padrão; a difere significativamente do grupo alto nível $(p .<0.05) t$-student Test. b difere significativamente do grupo baixo e moderado nível $(p .<0.05) t$-student Test.

A Tabela 3 apresenta os dados relativos ao padrão de consumo de substâncias no último mês pelos 3 grupos. Observou-se um percentual significativamente maior de não usuários de substâncias entre o grupo de baixo nível de atividade física ( $70 \%$ ), e um percentual significativamente maior de dependentes no grupo de atividade física alta (16\%). Não se observou diferenças significantes entre padrão de consumo e gênero, entre os grupos.

Tabela 3 - Padrão de consumo de substâncias dos adolescentes, considerando o gênero e o nível de atividade física. Dados expressos em porcentagem (n=754). Guarulhos, SP, Brasil, 2014/2015.

\begin{tabular}{|c|c|c|c|c|c|c|}
\hline \multirow[t]{2}{*}{$\begin{array}{c}\text { Padrão de } \\
\text { consumo }\end{array}$} & \multicolumn{2}{|c|}{$\begin{array}{c}\text { Baixo }^{a} \\
(\mathrm{~N}=247)\end{array}$} & \multicolumn{2}{|c|}{$\begin{array}{l}\text { Moderado }^{b} \\
(\mathrm{~N}=287)\end{array}$} & \multicolumn{2}{|c|}{$\begin{array}{c}\text { Alto }^{c} \\
(\mathrm{~N}=120)\end{array}$} \\
\hline & Homem & Mulher & Homem & Mulher & Homem & Mulher \\
\hline Não usuário & $34(\mathrm{~N}=84)$ & $36(\mathrm{~N}=89)$ & $21(\mathrm{~N}=60)$ & $32(\mathrm{~N}=92)$ & $21(\mathrm{~N}=25)$ & $23(\mathrm{~N}=28)$ \\
\hline Uso experimental & $10(\mathrm{~N}=24)$ & $10(\mathrm{~N}=24)$ & $12(\mathrm{~N}=34)$ & $18(\mathrm{~N}=52)$ & $19(\mathrm{~N}=23)$ & $13(\mathrm{~N}=17)$ \\
\hline Uso abusivo & $4(\mathrm{~N}=10)$ & $4(\mathrm{~N}=10)$ & $5(\mathrm{~N}=15)$ & $8(\mathrm{~N}=22)$ & $2(\mathrm{~N}=2)$ & $6(\mathrm{~N}=7)$ \\
\hline Dependentes & $2(\mathrm{~N}=6)$ & $0(\mathrm{~N}=0)$ & $2(\mathrm{~N}=6)$ & $2(\mathrm{~N}=6)$ & $8(\mathrm{~N}=9)$ & $8(\mathrm{~N}=9)$ \\
\hline
\end{tabular}

$a=X 2=6,1 ; p=0,1 ; \quad b=X 2=0,5 ; p=0,9 ; \quad c=X 2=3,8 ; p=0,2$

A Tabela 4 apresenta o consumo de substâncias psicotrópicas no último mês entre os três grupos, considerando o gênero. Observou-se maior percentual de uso das substâncias avaliadas (Exceto cocaína ou crack) entre os adolescentes do grupo de atividades físicas de alta intensidade (Com maior consumo entre os meninos), em comparação aos demais grupos. $\mathrm{O}$ grupo de atividade física moderada se diferenciou do grupo de intensidade baixa, apresentando maior percentual de usuários de energéticos e tabaco. Com relação ao gênero, foi constatado maior percentual de meninos do grupo de alta intensidade fazendo uso de bebidas alcoólicas; maior percentual de meninos do grupo de intensidade moderada e baixa fazendo uso de energéticos e maior percentual de meninas no grupo de intensidade moderada fazendo uso de tabaco.

Tabela 4 - Relação entre consumo de substâncias psicotrópicas no último mês e nível de atividade física, considerando o gênero. Valores expressos em porcentagem ( $n=754)$. Guarulhos, SP, Brasil, 2014/2015.

\begin{tabular}{|c|c|c|c|c|c|c|c|c|c|}
\hline \multirow[b]{2}{*}{ Substância } & \multicolumn{3}{|c|}{ Baixo (N=247) } & \multicolumn{3}{|c|}{ Moderado $(\mathrm{N}=287)$} & \multicolumn{3}{|c|}{ Alto $(N=120)$} \\
\hline & $\mathrm{H}$ & M & $\begin{array}{r}\text { Total } \\
(\%)\end{array}$ & $\mathrm{H}$ & M & $\begin{array}{r}\text { Total } \\
(\%)\end{array}$ & $\mathrm{H}$ & $M$ & $\begin{array}{r}\text { Total } \\
(\%)\end{array}$ \\
\hline Energéticos & $45,2 a$ & 27,0 & 36 & $60,0 a b$ & 52 & $55 b$ & 56,5 & $55,0 a$ & $56 a$ \\
\hline Álcool & 36,5 & 28,0 & 32 & 45,5 & 41,5 & $43 d$ & $66,5 a b c$ & 50,0 & $58 d$ \\
\hline Tabaco & 15,0 & 11,5 & 13 & 16,0 & $26,5 a$ & $22 b$ & $26,5 \mathrm{bc}$ & $25,0 \mathrm{~b}$ & $26 b$ \\
\hline Maconha & 11,0 & 11,5 & 7 & 11,5 & 9,0 & 10 & 16,5 & $17,0^{\mathrm{b}}$ & $17^{b c}$ \\
\hline
\end{tabular}


Tabela 4 - continuação

\begin{tabular}{|c|c|c|c|c|c|c|c|c|c|}
\hline \multirow{3}{*}{ Substância } & \multicolumn{3}{|c|}{ Baixo $(N=247)$} & \multicolumn{3}{|c|}{ Moderado $(\mathrm{N}=287)$} & \multicolumn{3}{|c|}{ Alto $(\mathrm{N}=120)$} \\
\hline & & & Total & & & Total & & & Total \\
\hline & $\mathrm{H}$ & M & $(\%)$ & $\mathrm{H}$ & M & $(\%)$ & $\mathrm{H}$ & M & $(\%)$ \\
\hline Inal ou Solventes & 2,5 & 1,5 & 2 & 8,0 & 9,0 & 8 & $15,0^{\mathrm{bc}}$ & $17,0^{\mathrm{bc}}$ & $16^{\mathrm{bc}}$ \\
\hline Ergogênicos & 2,5 & 0,8 & 2 & 7,5 & 4,0 & 5 & $13,5 \mathrm{bc}$ & $11,5^{b c}$ & $12^{\mathrm{bc}}$ \\
\hline Cocaína ou Crack & 0,8 & 0 & 0,4 & 3,0 & 0 & 1 & 2,5 & $8,5^{\mathrm{bc}}$ & $6^{\mathrm{bc}}$ \\
\hline
\end{tabular}

a difere do sexo oposto na mesma categoria;

b difere significativamente do grupo nível baixo $(p<0,05)$;

c difere significativamente do grupo nível moderado $(p<0,05)$;

difere dos demais grupos $(p<0,05)$;

\section{Discussão}

Este estudo objetivou trazer o tema à reflexão e avaliar a real contribuição das práticas de atividades físicas como método de prevenção ao uso de drogas considerando os índices de qualidade de vida, padrões de consumo e tipos de substâncias consumidas. Sabese que, para uma grande parte dos adolescentes, a entrada na puberdade e na adolescência é fonte de angústia e preocupação quanto a imagem corporal. Por esta razão a busca por atividades físicas nesta fase, independente do sexo, tende a crescer e tem por finalidade a melhora ou alteração da aparência física. ${ }^{(17)}$, o que podem explicar, ao menos em parte, o maior percentual de meninas presentes no grupo de atividade física moderada.

Essa busca por um corpo esteticamente perfeito tem levado muitos adolescentes a usar de forma precoce, abusiva e inadequada, substâncias as quais acreditam proporcionar rapidamente essa mudança estética. Dentre essas substâncias destacam-se os ergogênicos, cujo consumo tem aumentado consideravelmente entre adolescentes praticantes de atividades físicas ${ }^{(18)}$, e que no presente estudo, foram significativamente mais consumidas pelos meninos.

Pode-se inferir que o elevado consumo de substâncias ergogênicas deva-se ao fato da indústria lançar constantemente produtos com promessas de efeitos imediatos e milagrosos, associado a ausência de legislação rigorosa, que permite a comercialização sem prescrição médica. Esse descontentamento com a própria imagem pode induzir os adolescentes a buscar atividades físicas em alta intensidade, não somente com fins competitivos, mas também como valorização do corpo e da aparência, a serem atingidos muitas das vezes a qualquer custo.

Neste sentido, vale mencionar que muitos ergogênicos induzem a queima de aminoácidos, podendo provocar níveis elevados de cetose, acidez e amônia os quais, a longo prazo, podem gerar sobrecarga renal, desidratação e problemas cardiovasculares $^{(19)}$. No presente estudo, $12 \%$ dos adolescentes do grupo com alto nível de atividade física, em média com 15 anos de idade, fizeram consumo destas substâncias nos 30 dias que antecederam a coleta dos dados. Pode-se inferir que, devido a alta quantidade de atividade física praticada por aqueles adolescentes, este consumo tenha sido sugerido por um treinador ou colega que tenha feito uso. O que demonstra a necessidade emergencial de disseminar informações relativas ao perigo do consumo destas substâncias, em especial na adolescência.

Semelhantemente preocupante tem sido o consumo elevado de bebidas energéticas, não só no contexto recreativo, mas também no contexto esportivo. Por serem repositores metabólicos, leigamente, são considerados benéficos e desprovidos de qualquer malefício, justificando seu consumo descontrolado ${ }^{(14)}$

No presente estudo, quantidade expressiva de adolescentes que fizeram uso de bebidas energéticas (Baixo; 36\%; Moderado; $55 \%$ e Alto $56 \%$ ), muito provavelmente na expectativa de atingir melhor desempenho na prática esportiva. No entanto, pouco se sabe sobre o efeito do consumo de energéticos tanto no aumento do desempenho esportivo, quanto no aumento da capacidade física como resistência aeróbia, velocidade, força e potência muscular ${ }^{(20)}$.

Outro dado importante verificado neste estudo refere-se a prática de atividade física e qualidade de vida dos adolescentes. A Organização Mundial da Saúde menciona a atividade física, e prática de esportes em geral, como essenciais para a saúde e bem estar do ser humano ${ }^{(21)}$. Partindo deste princípio, muitas pessoas passaram a dedicar-se à prática esportiva além de níveis considerados benéficos. Neste sentido, muitos pais, ansiando por oferecer uma qualidade de vida diferenciada aos filhos, acabam expondo-os a níveis exacerbados de atividade física, comprometendo a vida social, familiar e acadêmica destes $^{(22)}$. 
Essa pesquisa, observou-se que os adolescentes do grupo com alto nível de atividade física apresentaram significativamente menores índices de qualidade de vida nas seguintes dimensões: autonomia ou relacionamento com os pais, sociabilidade ou relacionamento com amigos e desempenho escolar. Em outras palavras, pode-se verificar que devido ao fato destes adolescentes estarem demasiadamente envolvidos com o esporte, várias áreas da vida estavam sendo comprometidas. Sabe-se que jovens cujo envolvimento com esportes atinge níveis intensos, em geral, participam de competições nacionais e internacionais e, consequentemente, dedicam-se a treinos mais rigorosos e frequentes. Sendo assim, estes jovens acabam tendo maiores níveis de absenteísmo na escola, culminando em menor desempenho acadêmico, menor sociabilidade (Pois não desenvolvem vínculos de amizade), menor autonomia e relacionamento com os pais (Uma vez que estão sempre envolvidos em treinos e viagens) $)^{(23)}$.

Quanto a relação entre atividade física e uso de drogas, vale ressaltar que durante muito tempo creditou-se demasiadamente, à atividade física ou esportiva, propriedades preventivas ao uso de drogas ${ }^{(22)}$. Neste estudo, observou-se que, inversamente ao senso comum, os adolescentes do grupo de alto nível de atividade física apresentaram elevado consumo de várias substâncias (álcool, inalantes, maconha, tabaco, energéticos e ergogênicos). No mesmo sentido, alguns autores avaliaram a relação entre a prática esportiva e consumo de álcool ou tabaco de 13.872 estudantes entre 14 e 18 anos das 27 capitais brasileiras, e encontraram percentuais significativamente maiores de consumo de álcool entre os estudantes que praticavam regularmente esportes como, ginástica, futebol e musculação(24).Análogo a essas descobertas, estudo com universitários esportistas regulares, constataram que $13 \%$ deles haviam consumido maconha previamente a realização de atividades físicas, com vistas ao aumento do desempenho(10). Isto evidencia que as atividades físicas ou esportivas não podem ser consideradas prioritariamente fator de proteção para o uso de drogas, conforme afirmado pelode maneira geral . Tampouco pode-se afirmar que baixos níveis de atividades físicas ou esportivas ou a ausência destas, representem fator de risco para o uso de substâncias.

Um dado não esperado, porém importante, refere-se à porcentagem (70\%) de "não usuários" entre os adolescentes do grupo de baixo nível de atividade física. Assim, possivelmente, a qualidade de vida referente a dimensão familiar, percebida pelo adolescente e, representada pela interação entre ele e seus pais, da mesma maneira que sua sensação de pertencimento e sentir-se amado na família, seja o aspecto preponderante para explicar o elevado percentual desses "não usuários" atuando como fator de proteção para o uso de drogas. Além disso, como muitos destes adolescentes possuem dificuldades na escola, compreensão maior e melhor do uso de substâncias pelos educadores poderia fortalecer a proteção para o uso ${ }^{(25)}$.Indo além, pode-se observar que a ausência da prática de atividades físicas ou o baixo nível praticado, pouco representou para o desfecho de "não uso de drogas", quando comparado às variáveis relativas a qualidade de vida familiar e com amigos.

\section{Considerações Finais}

Diante dos resultados do presente estudo relativos a qualidade de vida familiar, social e escolar dos adolescentes, surge a preocupação sobre o impacto que as práticas desportivas intensas exercem na qualidade de vida destes. Verificou-se que os artigos existentes sobre qualidade de vida de adolescentes ou jovens esportistas, aborda a temática em senso lato, reforçando a importância da prática esportiva na qualidade de vida de modo geral. Porém não foram encontrados estudos referentes ao impacto da atividade física e esportiva sob dimensões específicas da qualidade de vida em adolescentes, tal qual realizado no presente estudo.

Em suma, considerada pela sociedade como um tema sempre associado a efeitos positivos, a prática de atividades físicas como método de prevenção ao uso de substâncias tornou-se uma afirmação pouco contestada, mesmo nos meios acadêmicos. Desta forma, sua real contribuição como método preventivo, em especial na adolescência, precisa ser repensada. O presente estudo apresenta algumas limitações: a) Amostra não representativa da população de adolescentes; b) O IPAQ fornece a classificação dos níveis de atividade física, porém não avalia os diferentes esportes.

Os resultados aqui encontrados propõem associação entre o nível de intensidade da atividade física e uso de drogas, e em hipótesealguma estabelece causalidade entre os fatos. Assim, sugere-se a necessidade emergencial de implementar programas de prevenção sistematizados, especificamente para a população adolescente, voltados a prevenção 
do consumo de substâncias em geral, aliada ao fornecimento de informações aos treinadores e técnicos a respeito do uso de bebidas energéticas e substâncias ergogênicas. Paralelamente, faz-se inevitável a reflexão sobre meios de se advertir os pais, os -familiares ou osresponsáveis acerca do impacto que altos níveis de intensidade esportiva podem acarretar na vida do adolescente, em especial no que refere-se a qualidade de vida relativa ao relacionamento familiar, social e acadêmico. Obviamente que não se pretende restringir, tampouco inibir, a prática esportiva competitiva entre adolescentes. Ao invés disto, propõe-se alertar os responsáveis (Treinadores e familiares) para que estes estejam atentos às diferentes esferas da vida do adolescente, que devem ser priorizadas tanto quanto a atividade esportiva.

\section{Referências}

01. Seabra FM, Mendonça MA, Thomis LA, Anjos JAM. Determinates biológicos e sócio-culturais associados à prática de atividade física de adolescentes. Cad Saúde Pública. 2008;24(4):721-36.

02. De Micheli D, AndradeALM, Silva EAD, Souza-Formigoni MLO. Drug Abuse in Adolescence: Neurobiological, Cognitive, and Psychological Issues. Switzerland: Springer International Publishing; 2016. 200 p.

03. Barros R. Os adolescentes e o tempo livre: lazer atividade física. In: Coates V, Françoso LA, Beznos GW. Medicina do adolescente. São Paulo: Sarvier; 1993.

04. Nazar-Beutelspacher A, Tapia-Conyer R, VillaRomero A, León-Alvarez G, Medina-Mora, ME, Salvatierra-Izaba, B. Factores asociados al consumo de drogas en adolescentes de áreas urbanas de México. Salud Publica Mex. 1994;36:46-54.

05. Wallander JL, Schmitt M, Koot HM. Quality of life measurement in children and adolescents: issues, instruments, and applications. J Clin Psychol. 2001;57(4):571-85.

06. Ravens-Sieberer U, Gosch A, Rajmil L, Erhart M, Bruil J, Duer W, Auquier P, et al. KIDSCREEN-52 qualityof-life measure for children and adolescents. Expert Rev Pharmacoecon Outcomes Res. 2005;5(3):353-64.

07. Barbosa D. O adolescente e o esporte. In: Maakaroun MF, Souza RP, Cruz AR, editores. Tratado de adolescência: um estudo multidisciplinar médica. Rio de Janeiro: Cultura; 1991. p. 128-35.

08. Valois RF, Mckeown RE, Saunders RP, Pate RR, Winnaill SD. Relationship between physical activity highschool adolescents. J Sch Health. 1995;65:438.
09. Dearwater SR, Anderson R, Olsen T, Kriska AM, Laporte RE, Aaron DJ. Physical activity and the initiation of high-risk health behaviors in adolescents. Med Sci Sports Exerc. 1995;27:39-45.

10. Lorente FO, Souville M, Griffet J, Grélot L. Participation in sports and alcohol consumption among French adolescents. Addict Behav. 2004; 29(5):941-6.

11. Matsudo S, Araújo T, Marsudo V, Andrade D, Andrade E, Braggion G. Questinário internacional de atividade f1sica (IPAQ): estudo de validade e reprodutibilidade no Brasil. Rev Bras Ativ Fís Saúde. 2001;6(2):5-18.

12. Hallal $P$, Siqueira FV. Compliance with vigorous physical activity guidelines in Brazilian adults: prevalence and correlates. J Phys Act Health. 2004;1(4):389-900.

13. Pollock ML, Gaesser GA, Butcher JD, Després JD, Dishman RK, Franklin BA, et al. (American College of Sports Medicine. Position stand: the recommended quantity and quality of exercise for developing and maintaining fitness in healthy adults. Med Sci Sports Exerc. 1998;30(5):975-91.

14. Coelho-Ravagnani CDF, Melo FCL, Ravagnani FCP, Burini FHP, Burini RC. Estimativa do equivalente metabólico (MET) de um protocolo de exercícios físicos baseada na calorimetria indireta. Rev Bras Med Esporte. 2013;19(2):134-8.

15. Guedes DP, Guedes JERP. Tradução, adaptação transcultural e propriedades psicométricas do KIDSCREEN-52 para a população brasileira. Rev Paul Pediatr. 2001;29(3):364-71.

16. De Micheli D, Fisberg M, Formigoni MLO. Estudo da efetividade da intervenção breve para o uso de álcool e outras drogas em adolescentes atendidos num serviço de assistência primária à saúde. Rev Assoc Med Bras. 2004;50(3):305-13.

17. Ribeiro P, Oliveira P. Culto ao Corpo: beleza ou doença? Adolesc Saude. 2001; 8(3):63-9.

18. Harrison R, Holt D, Pattison DJ, Elton PJ. Are those in need taking dietary supplements? A survey of 21.923 adults. Br J Nutr. 2004;9(4):617-23.

19. Poortmans J, Francaux M. Adverse efects of creatine supplementation. Rev Bras Med Esporte. 2000;30(3):15570.

20. Longenecker G. Como agem as drogas: abuso de drogas e o organismo humano. São Paulo: Editora Quark do Brasil; 1998.

21. World Health Organization (WHO). Health and development through physical activity and sport. Switzerland: WHO; 2003.

22. Terry-McElrath YM, O'Malley PM, Johnston LD. Exercise and substance use among American youth, 1991-2009. Am J Prev Med. 2011;40:530-40. 
23. Bedendo A, Opaleye E, Andrade ALM, Noto AR. Heavy episodic drinking and soccer practice among high school students in Brazil: the contextual aspects of this relationship. BMC Public Health. 2013;13:1-8.

24. Bedendo A, Noto AR. Sports practices related to alcohol and tobacco use among high school students. Rev Bras Psiquiatr. 2015;37(2):99-105.

25. Souza FBD, Andrade ALM, Rodrigues TP, Nascimento MO, De Micheli D. Avaliação das concepções de educadores de escolas públicas e particulares sobre uso de drogas: um estudo exploratório. Estud Pesqui Psicol. 2015;15(3):1081-95. 